\title{
Neuroimaging clues of migraine aura
}

\author{
Nouchine Hadjikhani ${ }^{1} 2^{*}$ (D) and Maurice Vincent ${ }^{3}$
}

\begin{abstract}
While migraine headaches can be provoked, or predicted by the presence of an aura or premonitory symptoms, the prediction or elicitation of the aura itself is more problematic. Therefore, imaging studies directly examining the aura phenomenon are sparse. There are however interictal imaging studies that can shed light on the pathophysiology of the migraine with aura (MWA) cascade. Here, we review findings pointing to the involvement of cortical spreading depression (CSD) and neuroinflammation in MWA. Whether asymptomatic CSD also happens in some migraine without aura is still under debate. In addition, new evidence points to glial activation in MWA, indicating the involvement of astrocytes in the neuroinflammatory cascade that follows CSD, as well as dural macrophages, supporting the involvement of the trigeminovascular system in migraine pain.
\end{abstract}

Keywords: (3-10): Migraine, Aura, CSD, Brain imaging

\section{Background}

\section{The symptom spectrum of the migraine aura}

Migraine, a partially genetic prevalent brain disorder [1], presents with attacks separated by interictal phases. An attack can be considered as a dysfunctional brain state probably related with long-lasting anatomical and functional consequences, as shown by our group and others [2, 3].

In at least a third of migraine attacks, the headache phase is preceded and/or accompanied by transient neurological symptoms referred to as aura. In this case, the condition is classified as migraine with aura (MWA), contrary to migraine without aura (MWoA), when no aura symptom is present [4]. The most frequent aura phenotype consists of positive and/or negative visual phenomena, present in up to $99 \%$ of the patients [5]. Phenotypes include primary visual disturbances, for example flashes of light, moving zigzags expanding in a horseshoe pattern, white spots, "like looking through air close to a road paved with asphalt in a very hot sunny day", colored spots; or less frequent more complex perceptions, such as fractured vision, dysmorphopsias, lilliputian (too small) or brobdingnagian (too large) hallucinations, misperceptions of distances, hemianopsias,

\footnotetext{
* Correspondence: nouchine@nmr.mgh.harvard.edu

${ }^{1}$ A.A. Martinos Center for Biomedical Imaging, Massachusetts General Hospital, Harvard Medical School, Boston, USA

${ }^{2}$ Gillberg Neuropsychiatry Center, Sahlgrenska Academy, Gothenburg

University, Gothenburg, Sweden

Full list of author information is available at the end of the article
}

tunnel vision, among others [6]. Since visual auras varies in form, severity, and duration, the anatomical location, extent, and probably nature of the underlying occipital dysfunction must vary accordingly.

Auras can present also with sensory (mostly paraesthesias in an upper limb and/or hemiface) or language (mainly paraphasia and anomic aphasia) disturbances, either isolated or (more frequently) in combination with visual phenomena, meaning that other brain structures than those related to visual processing can be affected. When aura involves motor weakness, patients are classified as either sporadic or familial hemiplegic migraine [4]. The underlying dysfunction can affect different areas of the cortex consecutively, as successive aura symptoms can build-up over the course of several minutes. In conclusion, there is a striking inter- and intra-patient variability regarding the presence, nature, pattern, and timing of aura symptoms in migraine [6].

The ICHD-3 defines aura as fully-reversible visual, sensory or other central nervous system symptoms that usually develop gradually in the context of a migraine attack. Three out of 6 features are required: 1) at least one aura symptom spreads gradually over $\geq 5 \mathrm{~min}$; 2) two or more aura symptoms occur in succession; 3 ) each individual aura symptom lasts 5-60 min; 4) at least one aura symptom is unilateral; 5) at least one aura symptom is positive; and 6) the aura is accompanied, or followed within $60 \mathrm{~min}$, by headache. Aura types are visual, sensory, speech and/or language, motor, brainstem, and 
retinal. Typical aura consists of visual, sensory and/or speech/language symptoms by definition [4].

The underlying transitory dysfunction that causes aura can theoretically affect any region of the brain. Because symptoms originated in some areas may be less clear, it is possible some aura-like phenomena apart from typical symptoms do not fulfill the ICHD-3 aura criteria. While visual cortex excitation can be translated into complex and/or intense visual phenomena, it is unclear how other visual phenomena emerge. Likewise, little is known about how possibly aura-related cortical dysfunction would be translated if occurring in areas where neurological signs could be relatively subtle, such as the prefrontal cortex for example. Paucisymptomatic or "symptom-free aura" (i.e., the occurrence of the pathophysiological mechanisms underlying aura but in the absence of symptoms) happening in visual as well as other parts of the brain would lead to the diagnosis of migraine without aura (MWoA). The existence and frequency of "symptom-free aura" in MWoA and MWA are crucial yet unanswered questions. A precise distinction between aura and migraine-related cortical symptoms from distinct pathophysiology cannot yet be established.

Cortical spreading depression (CSD), a slow, selfpropagating wave of neuronal and glial depolarization is the neurophysiological phenomenon most likely involved with the pathophysiology of the migraine aura [7, 8], (for reviews see $[9,10])$. It is noteworthy that different classes of migraine preventive medications tested chronically dose-dependently reduced the CSD frequency and increased the CSD elicitation threshold [11].

In a study published in 2007 [12], we sent questionnaires to more than 500 migraine patients asking for the presence of symptoms such as difficulties in recognizing faces, colors, the presence of language or memory abnormalities, and other cognitive changes. Remarkably, while $72.2 \%$ of MWA patients reported such symptoms, $48.6 \%$ of MWoA patients also described symptoms that could be attributed to cortical dysfunctions, potentially linked to cortical spreading depression (CSD) phenomena happening in higher tier areas. In line with common clinical sense, we concluded that neurological symptoms other than the classic aura may be underestimated in migraineurs. It is possible that at least part of these symptoms are produced by CSD-like phenomena in relatively silent areas such as the frontal lobe, where symptoms remain unapparent until lesions grow to a large degree [13]. In support of this possibility are the very recently reported changes in speech patterns prior to migraine attack in individuals diagnosed with migraine without aura, present in close to half of the patients during a migraine attack [14], showing that other periictal symptoms may be more common than previously thought.
Aura and the various components of the migraine attack Migraine has different phases, not necessarily evident in all patients - premonitory, aura, headache and postdromic [15]. Evidence suggests that migraine is of neurogenic origin, and that the related vascular events are consequences of neuronal changes [8, 16-19]. Daily imaging of a MWoA patient for 30 consecutive days showed hypothalamic activation as early as $24 \mathrm{~h}$ before the migraine pain, suggesting that the hypothalamus could be a generator of migraine attack [20]. Although migraine attacks depend on a susceptible brain, the relative independence between their different components suggests that, rather than a rigid successive chain of events, the migraine episode can be better explained sometimes as a net dysfunction, possibly activated from different places and sequences. Accordingly, the pain may vary in location, the aura can be present or not and start after the pain, the premonitory symptoms can vary, and so on. The aura-headache coupling is particularly puzzling [21-23]. Aura is neither ubiquitous nor phenotypically uniform, even in a single patient. Knowledge about migraine phenotypes and pathophysiology supports the concept that both patterns are possible and not mutually exclusive: a migraine attack may the result of a successive series of events where one necessarily triggers the next, or it may behave as a network of possible brain dysfunctions disrupted in different combinations or sequences.

\section{Imaging the migraine aura}

Key questions involve aura neuroimaging. Are there changes in the brain that accurately reflect the occurrence of aura symptoms? Is the aura CSD hypothesis supported by neuroimaging? If so, are these CSD-like neuroimaging hallmarks present exclusively when aura symptoms are reported? How aura-associated neuroimaging data couple with imaging hallmarks of other migraine attacks phases? And finally, are there detectable changes in the brain that either predispose or appear as a result of migraine aura?

The first report of transient hyperemia followed by spreading decreased cerebral blood flow was described in 1981 by Olesen et al. [24]. They used intra-arterial Xenon133 to show that the concept of migraine aura as a vasospastic event was erroneous (see also [25]). Subsequent studies by the Danish group found results suggestive of spreading oligemia $[26,27]$ in MWA. It became natural to suppose that the spreading nature of the circulatory changes, mirroring the $2-3 \mathrm{~mm} / \mathrm{min}$ pace of CSD and not respecting the supply territories of large arteries, would somehow correspond to the CSD phenomenon [7].

In our seminal study $(\mathrm{NH})$ [8], we reported the case of a patient who complained of having migraine auras 
every time after he had been playing basketball for a sustained amount of time. We therefore decided to invite him to play at the sports facilities located right next to the imaging center, for as long as he thought would be necessary to trigger a migraine aura after stop playing, at which point we would go to the MRI center and image him, showing periods of $32 \mathrm{~s}$ of flickering radial checkerboard alternating with $32 \mathrm{~s}$ of a black screen with a fixation cross. The participant had a squeeze ball he would press when the aura would start, and when it would end. We repeated this a couple of times, and we also acquired interictal data in order to ensure that we would get a stable signal using this protocol of on/off visual stimulation for a sustained amount of time. Using these datasets, we were able to demonstrate signal changes that contained at least eight characteristics in common between the observed events and animal CSD studies. The fMRI signal in the occipital cortex was time-locked to the onset of the visual aura, the quality of the aura was consistent with the function of the area where it started (area V3a), and its propagation was congruent with the retinotopy of the visual percept. The initial focal BOLD signal increase possibly reflected vasodilation; it progressed contiguously and slowly over the occipital cortex to affect adjacent voxels. After this initial increase, the BOLD signal diminished, (possibly reflecting vasoconstriction following the initial vasodilation), and was then suppressed transiently. During periods with no visual stimulation other than a fixation cross, but during which the subject was experiencing scintillations, the BOLD signal change followed the retinotopic progression of the visual percept, moving from the center of the visual field towards the periphery. We concluded from this study that the data strongly suggested that a propagating event akin to CSD generates the migraine visual aura in human visual cortex.

Clinically, both MWA and MWoA share the same attack features except for aura. They are defined by the same pain characteristics: unilateral, pulsating, and aggravated by physical activity pain lasting $4-72 \mathrm{~h}$, associated with the same symptoms and signs (e.g. changes in appetite, nausea, vomiting, light and noise sensitivity). In addition, they can both be triggered by the same substances (e.g., Nitric Oxide releasers or some neuropeptides) [28], and respond to the same preventive or therapeutic treatments. Symptomatic aura is obviously not obligatory prior to the migraine pain, otherwise neither MWoA nor MWA patients having aura symptoms starting after the headache phase would be possible. In about $20 \%$ of cases the headache starts before aura or simultaneously with it [21].

There is evidence to suggest that cortical dysfunction, possibly involving CSD-like phenomena, is also present in migraine without aura [12, 24, 29-31]. Thus, CSD at the same or other cortical sites could be either asymptomatic, evoke typical aura phenomena, or give rise to symptoms not classically recognized as aura. Studies confirming the presence of CSD-like neuroimaging hallmarks in other less typical migraine-generated transitory cortical dysfunctions are not available, although evidence supports the presence of CSD-like phenomena in migraine without aura. As an example, Woods et al. [29] reported in 1994 spreading cerebral hypoperfusion in a patient suffering from migraine without aura, providing the first evidence that CSD may also underlie MWoA although one should mention that the patient in the report by Woods actually experienced transient visual symptoms (hazy vision). Admitting that CSD is the substrate of aura and possibly occurs in migraine with atypical, subtle, or no aura symptom, the possibility exists that some MWoA patients have the headache associated - or even induced - by relatively silent CSD.

It is noteworthy that ca. 17\% of MWA patients may present prolonged episodes [32]. Neuroimaging evidence linking this aura phenotype to CSD-like changes in humans is lacking. In the $\mathrm{KCl}$ stimulated gyrencephalic feline cortex, secondary CSD events were shown to propagate in parallel to the gyrus in which they took place originally, encompassing a significantly smaller cortical area and propagating with a significantly slower velocity [33]. It remains to be demonstrated whether secondary or parallel CSD waves in the human cortex could manifest as prolonged aura in migraineurs. The fact that migraine aura is characterized by symptoms that can start in succession (with or without a symptomfree interval) or simultaneously indicated that either multiple CSD waves arise at different points in topography and time, or waves may travel asymptomatically across cortical areas producing later symptoms at regions distant from the cortex originally symptomatic [6].

CSD is accompanied by matrix metalloproteinase- 9 (MMP-9) activation and neurogenic inflammation [34, 35]. Indirect evidence of neuroinflammation has been shown in migraine, with intracranial plasma extravasation (one case report, [36]), and gadolinium enhancement (one case report, [37]). Despite a lot of efforts, it has been difficult to reliably demonstrate gadolinium enhancement in migraine. Some studies have used superparamagnetic iron oxide nanoparticles (ferumoxytol) to assess areas of blood brain barrier (BBB) dysfunction during neuroinflammation [38]. These particles are taken up by many elements of the immune system, including the microglia - however, their size comparable to viruses, makes them less likely to cross the BBB, supposedly intact in MWoA [39] and MWA [40], although increased levels of matrix metalloproteinase-9 (MMP-9) have been associated with migraine [41, 42]. It is possible that the techniques used so far have not been 
sensitive enough to definitely objectify BBB disruption in migraine.

Animal models of CSD indicate that neuroinflammation and microglia activation may be key factors in the generation of the pain associated with migraine [43, 44]. A study in rodents has demonstrated that a complex cascade induced by CSD leads to neuroinflammation, including astrocytic and microglia activation [45], eventually leading to pain via activation of the trigeminovascular system. That study demonstrated that CSD leads to Pannexin 1 channels opening and activation of pro-inflammatory mediators, that in turn induce cyclooxygenase- 2 and inducible Nitric Oxide synthase expression in astrocytes, and microglia activation. Astrocyte release of cytokines, prostanoids and Nitric Oxide in the subarachnoid space promote sustained activation of the trigeminal nerve fibers surrounding pial vessels, and the trigeminal nerve collaterals innervating the middle meningeal artery initiate neurogenic inflammation, including mast cells degranulation.

We recently used combined PET/MR imaging techniques with [11C]PBR28, a radioligand that binds to the $18 \mathrm{kDa}$ translocator protein, a marker of glial activation, in migraineurs with visual aura who had experienced one or more migraine episodes during the two weeks preceding the imaging session [46]. We observed increased signal in areas previously shown to be involved in CSD generation (primary visual cortex, visual areas V3A and MT, and Broca's area) as well as with areas involved in pain processing (thalamus and primary/secondary somatosensory and insular cortices). Increase of the signal was also positively associated with the frequency of migraine attacks. We also found increased signal in the frontal pole and the orbitofrontal cortex, areas in which CSD may produce symptoms that may be difficult to notice or to interpret. In that same group of patients, we also observed increased uptake of [11C]PBR28 in the meninges, possibly reflecting activation of dural macrophages following MWA (Hadjikhani et al., in preparation).

In migraine, several studies have pointed to neuroinflammation as a possible substrate for the generation of pain $[47,48]$, after the release of proinflammatory peptides subsequent to CSD [34].

CSD has been shown to induce pial vasodilation involving the release of CGRP [49]. The CSD-related delayed hyperemia is abolished by transecting trigeminal and parasympathetic fibers [34]. Olcegepant and other small molecule CGRP antagonists, as well as CGRP and CGRP receptor antibodies have shown to modulate CSD [50]. On the other hand, the CGRP antagonist BIBN4096 did not block in rats the CSD-induced activation of meningeal afferents, suggesting that a CSD-evoked headache involves other mediators [51].
CGRP, a vasodilating neuropeptide peripherally released by trigeminal fibres, was found increased in the jugular blood ipsilaterally to the head pain during a migraine attack [52], and is reduced in parallel with headache lowering following sumatriptan treatment [53]. New effective anti-migraine treatments block CGRP signaling [54].

In the animal model, genetically driven cortical hyperexcitability predispose to CSD [55]. Hyperexcitability of the central nervous system has been speculated in both MWA and MWoA [56-58], and our group has reported microstructural changes in the thalamus, an important structure in the control of cortical excitability, in migraineurs [59].

Repetitive episodes of neuronal inflammation may result in retrograde degeneration of the neurovascular unit, with as a consequence small lesions in the brain [60]. Migraine has indeed been linked to silent infarct-like lesions (identified by magnetic resonance imaging (MRI) regardless of clinical manifestations) [61-63] that may be triggered by vascular changes linked with inflammation. However, in a follow-up of the original CAMERA study, MWA was not associated with white matter lesions [64], and a large population-based study with female twins did not confirm an increased risk of silent infarcts in migraine with aura [65]. Other structures seem to be affected by repetitive migraine attacks including the thalamus [59], the frontal lobe and the cerebellum [66]; and cortical thickness increases in the somatosensory cortex and in extrastriate visual areas have been reported [67-69]. Notably, Gaist et al. [70]. recently reported increased cortical thickness in a large group of MWA patients compared to controls, confirming our previous findings [68], but Hougaard et al. [71] found no increase of somatosensory cortical thickness in MWA patients with somatosensory aura, suggesting that structural changes (increased neuronal density?) in visual cortical areas predispose to CSD, but that CSD per se does not induce increased cortical thickness. In addition, our recent studies have also shown alterations of brain function, with increased connectivity in the pain matrix [72, 73]. It is also known that repetitive episodes of pain also lead to allodynia, reflecting hypersensitivity of the somatosensory system [74-76]. Whether long-term maladaptive plastic changes are at the basis of the chronification in migraine, which happens at a yearly rate of about 3\% needs to be further explored [77].

Other conditions such as occipital epilepsy can mimic migraine aura [78-80], although seizures tend to be shorter, occur in clusters, sometimes developing into temporal lobe or generalized epilepsy; however the differential diagnosis may be complicated by the fact that they can be accompanied by migrainous headache. 
Human neuroimaging depicting CSD-like phenomena in connection to seizures is lacking. The actual role of CSD in epilepsy is complex and obscure as data suggest that CSD can predispose to epileptic activity and vice-versa [81]. Migraine aura can be mistaken for a stroke [82, 83] as areas of hypoperfusion can be evidenced during migraine aura. Transient global amnesia (TGA), manifested as a transitory memory loss, could be a manifestation of hippocampal CSD, but may as well be due to a transient ischemic attack or a stroke, and abnormalities in diffusion weighted imaging can be observed due to either etiologies [84], rendering the differential diagnosis even more difficult.

Although not yet reproduced by similar studies, hypoperfusion of the cerebellum and crossed cerebellar diaschisis, an associated hypoperfusion of the frontal cortex in the opposite hemisphere, were reported in patients with MWA. Because it is not accompanied by severe diffusion imaging abnormalities nor cerebellar infarction, even in those who have prolonged symptoms for up to $24 \mathrm{~h}$, it may be considered a benign phenomenon [85].

\section{Conclusion}

In conclusion, neuroimaging data indicate that: 1) migraine aura is related to a CSD-like phenoma in MWA patients; 2) spreading phenomena similar to CSD may occur in MWoA, suggesting that either CSD can be asymptomatic, that symptomatic aura depends on other factors than just CSD, or that CSD in MWA differs fundamentally from the spreading oligoemia found in MWoA; 3) visual aura most probably starts at visual cortical areas such as V3A and MT; 4) glial activation is present following migraine attacks at areas previously shown to be involved with aura generation and/or pain processing, increasing in accordance to the headache frequency; 5) activation of meningeal macrophages in MWA further support the activation of the trigeminovascular system by CSD. It remains to be explained how the aura phenotype varies vastly even when the same cortical areas are involved; to which extent CSD happens in MWoA and what makes it phenotypically apparent, and how a CSD-like phenomenon as demonstrated by neuroimaging couples mechanistically with the other phases of the migraine attack.

\section{Abbreviations}

BBB: Blood brain barrier; BOLD signal: Blood-Oxygen-Level Dependent signal; CSD: Cortical spreading depression; fMRI: Functional Magnetic Resonance Imaging; MMP-9: Metalloproteinase-9; MWA: Migraine with aura; MWoA: Migraine without aura; PET: Positron Emission Tomography

\section{Acknowledgements}

N/A.

\section{Funding}

The article-processing charges for the article has been sponsored by the European Headache Federation.

\section{Availability of data and materials}

N/A.

\section{Authors' contributions}

$\mathrm{NH}$ and MV reviewed the literature, wrote the manuscript, and read and approved the final manuscript.

\section{Ethics approval and consent to participate}

N/A.

\section{Consent for publication}

N/A.

\section{Competing interests}

The authors declare that they have no competing interests.

\section{Publisher's Note}

Springer Nature remains neutral with regard to jurisdictional claims in published maps and institutional affiliations.

\section{Author details}

${ }^{1}$ A.A. Martinos Center for Biomedical Imaging, Massachusetts General Hospital, Harvard Medical School, Boston, USA. ${ }^{2}$ Gillberg Neuropsychiatry Center, Sahlgrenska Academy, Gothenburg University, Gothenburg, Sweden. ${ }^{3}$ Neuroscience Research, Eli Lilly and Company, Indianapolis, USA.

Received: 1 February 2019 Accepted: 14 March 2019

Published online: 03 April 2019

References

1. Anttila V, Winsvold BS, Gormley P, Kurth T, Bettella F, McMahon G, et al (2013) Genome-wide meta-analysis identifies new susceptibility loci for migraine. Nat Genet 45(8):912-917

2. Hougaard A, Amin FM, Ashina M (2014) Migraine and structural abnormalities in the brain. Curr Opin Neurol 27(3):309-314

3. May A (2017) Understanding migraine as a cycling brain syndrome: reviewing the evidence from functional imaging. Neurol Sci 38(Suppl 1): $125-130$

4. Headache Classification Committee of the International Headache Society (IHS) (2018) The international classification of headache disorders, 3rd edition. Cephalalgia 38(1):1-211

5. Russell MB, Olesen J (1996) A nosographic analysis of the migraine aura in a general population. Brain. 119(Pt 2):355-361

6. Viana M, Sances G, Linde M, Ghiotto N, Guaschino E, Allena M et al (2017) Clinical features of migraine aura: results from a prospective diary-aided study. Cephalalgia. 37(10):979-989

7. Lauritzen M (1994) Pathophysiology of the migraine aura. The spreading depression theory. Brain. 117(Pt 1):199-210

8. Hadjikhani N, Sanchez del Rio M, Wu O, Schwartz D, Bakker D, Fischl B et al (2001) Mechanisms of migraine aura revealed by functional MRI in human visual cortex. Proc Natl Acad Sci U S A 98(8):4687-4692

9. Schwedt TJ, Goadsby PJ (2010) 14th international headache congress: basic science highlights. Headache. 50(3):520-526

10. Lauritzen M, Dreier JP, Fabricius M, Hartings JA, Graf R, Strong AJ (2011) Clinical relevance of cortical spreading depression in neurological disorders: migraine, malignant stroke, subarachnoid and intracranial hemorrhage, and traumatic brain injury. J Cereb Blood Flow Metab 31(1):17-35

11. Ayata C, Jin HW, Kudo C, Dalkara T, Moskowitz MA (2006) Suppression of cortical spreading depression in migraine prophylaxis. Ann Neurol 59(4): 652-661

12. Vincent MB, Hadjikhani N (2007) Migraine aura and related phenomena: beyond scotomata and scintillations. Cephalalgia. 27(12):1368-1377

13. Maurice-Williams RS, Dunwoody G (1988) Late diagnosis of frontal meningiomas presenting with psychiatric symptoms. Br Med J (Clin Res Ed) 296(6639):1785-1786

14. Schwedt TJ, Peplinski J, Garcia-Filion P, Berisha V (2018) Altered speech with migraine attacks: a prospective, longitudinal study of episodic migraine without aura. Cephalalgia

15. Goadsby PJ, Holland PR, Martins-Oliveira M, Hoffmann J, Schankin C, Akerman S (2017) Pathophysiology of migraine: a disorder of sensory processing. Physiol Rev 97(2):553-622 
16. Bowyer SM, Aurora KS, Moran JE, Tepley N, Welch KM (2001) Magnetoencephalographic fields from patients with spontaneous and induced migraine aura. Ann Neurol 50(5):582-587

17. Cao Y, Welch KMA, Aurora S, Vikingstad E (1999) Functional MRI-BOLD of visually triggered headache in patients with migraine. Arch Neurol 56:548-554

18. Kaube H, Herzog J, Kaufer T, Dichgans M, Diener HC (2000) Aura in some patients with familial hemiplegic migraine can be stopped by intranasal ketamine. Neurology. 55(1):139-141

19. Shibata K, Osawa M, Iwata M (1998) Pattern reversal visual evoked potentials in migraine with aura and migraine aura without headache. Cephalalgia. 18(6):319-323

20. Schulte LH, May A (2016) The migraine generator revisited: continuous scanning of the migraine cycle over 30 days and three spontaneous attacks. Brain. 139(Pt 7:1987-1993

21. Viana M, Linde M, Sances G, Ghiotto N, Guaschino E, Allena M et al (2016) Migraine aura symptoms: duration, succession and temporal relationship to headache. Cephalalgia. 36(5):413-421

22. Viana M, Sances G, Ghiotto N, Guaschino E, Allena M, Nappi G et al (2016) Variability of the characteristics of a migraine attack within patients. Cephalalgia. 36(9):825-830

23. Hansen JM, Goadsby PJ, Charles AC (2016) Variability of clinical features in attacks of migraine with aura. Cephalalgia. 36(3):216-224

24. Olesen J, Larsen B, Lauritzen M (1981) Focal hyperemia followed by spreading oligemia and impaired activation of rCBF in classic migraine. Ann Neurol 9(4):344-352

25. Olesen J, Tfelt-Hansen P, Henriksen L, Larsen B (1981) The common migraine attack may not be initiated by cerebral ischaemia. Lancet. 2(8244): 438-440

26. Lauritzen M, Skyhoj Olsen T, Lassen NA, Paulson OB (1983) Changes in regional cerebral blood flow during the course of classic migraine attacks. Ann Neurol 13(6):633-641

27. Olesen J, Friberg L, Olsen TS, Iversen HK, Lassen NA, Andersen AR et al (1990) Timing and topography of cerebral blood flow, aura, and headache during migraine attacks. Ann Neurol 28(6):791-798

28. Ranson R, Igarashi H, MacGregor EA, Wilkinson M (1991) The similarities and differences of migraine with aura and migraine without aura: a preliminary study. Cephalalgia. 11(4):189-192

29. Woods RP, lacoboni M, Mazziotta JC (1994) Brief report: bilateral spreading cerebral hypoperfusion during spontaneous migraine headache. N Engl J Med 331(25):1689-1692

30. Sanchez del Rio M, Bakker D, Wu O, Agosti R, Mitsikostas DD, Ostergaard L et al (1999) Perfusion weighted imaging during migraine: spontaneous visual aura and headache. Cephalagia. 19:1-7

31. Ferrari MD, Haan J, Blokland JA, Arndt JW, Minnee P, Zwinderman AH et a (1995) Cerebral blood flow during migraine attacks without aura and effect of sumatriptan. Arch Neurol 52(2):135-139

32. Viana M, Sances G, Linde M, Nappi G, Khaliq F, Goadsby PJ et al (2018) Prolonged migraine aura: new insights from a prospective diary-aided study. J Headache Pain 19(1):77

33. Bockhorst KH, Smith JM, Smith MI, Bradley DP, Houston GC, Carpenter TA et al (2000) A quantitative analysis of cortical spreading depression events in the feline brain characterized with diffusion-weighted MRI. J Magn Reson Imaging 12(5):722-733

34. Bolay H, Reuter U, Dunn AK, Huang Z, Boas DA, Moskowitz MA (2002) Intrinsic brain activity triggers trigeminal meningeal afferents in a migraine model. Nat Med 8(2):136-142

35. Gursoy-Ozdemir Y, Qiu J, Matsuoka N, Bolay H, Bermpohl D, Jin $\mathrm{H}$ et al (2004) Cortical spreading depression activates and upregulates MMP-9. J Clin Invest 113(10):1447-1455

36. Knotkova H, Pappagallo M (2007) Imaging intracranial plasma extravasation in a migraine patient: a case report. Pain Med 8(4):383-387

37. Arnold G, Reuter U, Kinze S, Wolf T, Einhaupl KM (1998) Migraine with aura shows gadolinium enhancement which is reversed following prophylactic treatment. Cephalalgia. 18(9):644-646

38. Weinstein JS, Varallyay CG, Dosa E, Gahramanov S, Hamilton B, Rooney WD et al (2010) Superparamagnetic iron oxide nanoparticles: diagnostic magnetic resonance imaging and potential therapeutic applications in neurooncology and central nervous system inflammatory pathologies, a review. J Cereb Blood Flow Metab 30(1):15-35
39. Amin FM, Hougaard A, Cramer SP, Christensen CE, Wolfram F, Larsson HBW et al (2017) Intact blood-brain barrier during spontaneous attacks of migraine without aura: a 3T DCE-MRI study. Eur J Neurol 24(9):1116-1124

40. Hougaard A, Amin FM, Christensen CE, Younis S, Wolfram F, Cramer SP et al (2017) Increased brainstem perfusion, but no blood-brain barrier disruption, during attacks of migraine with aura. Brain. 140(6):1633-1642

41. Imamura K, Takeshima T, Fusayasu E, Nakashima K (2008) Increased plasma matrix metalloproteinase-9 levels in migraineurs. Headache. 48(1):135-139

42. Martins-Oliveira A, Speciali JG, Dach F, Marcaccini AM, Goncalves FM, Gerlach RF et al (2009) Different circulating metalloproteinases profiles in women with migraine with and without aura. Clin Chim Acta 408 (1-2):60-64

43. Cui Y, Takashima T, Takashima-Hirano M, Wada Y, Shukuri M, Tamura Y et al (2009) 11C-PK11195 PET for the in vivo evaluation of Neuroinflammation in the rat brain after cortical spreading depression. Nucl Med 50(11):1904-1911

44. Gehrmann J, Mies G, Bonnekoh P, Banati R, lijima T, Kreutzberg GW et al (1993) Microglial reaction in the rat cerebral cortex induced by cortical spreading depression. Brain Pathol 3(1):11-17

45. Karatas H, Erdener SE, Gursoy-Ozdemir Y, Lule S, Eren-Kocak E, Sen ZD et al (2013) Spreading depression triggers headache by activating neuronal Panx1 channels. Science. 339(6123):1092-1095

46. Albrecht D, Mainero C, Ichijo E, Ward N, Granziera C, Zurcher NR et al (2019) Imaging of neuroinflammation in migraine with aura - a [11C] PBR28 PET/ MR study. Neurology. in press

47. Markowitz S, Saito K, Moskowitz MA (1987) Neurogenically mediated leakage of plasma protein occurs from blood vessels in dura mater but not brain. J Neurosci 7(12):4129-4136

48. Moskowitz MA (1984) The neurobiology of vascular head pain. Ann Neurol 16(2):157-168

49. Wahl M, Schilling L, Parsons AA, Kaumann A (1994) Involvement of calcitonin gene-related peptide (CGRP) and nitric oxide (NO) in the pial artery dilatation elicited by cortical spreading depression. Brain Res 637 $(1-2): 204-210$

50. Close LN, Eftekhari S, Wang M, Charles AC, Russo AF (2019) Cortical spreading depression as a site of origin for migraine: role of CGRP. Cephalalgia. 39(3):428-434

51. Zhao J, Levy D (2018) The CGRP receptor antagonist BIBN4096 inhibits prolonged meningeal afferent activation evoked by brief local $\mathrm{K}(+)$ stimulation but not cortical spreading depression-induced afferent sensitization. Pain Rep 3(1):e632

52. Goadsby PJ, Edvinsson L, Ekman R (1990) Vasoactive peptide release in the extracerebral circulation of humans during migraine headache. Ann Neurol 28(2):183-187

53. Goadsby PJ, Edvinsson L (1993) The trigeminovascular system and migraine: studies characterizing cerebrovascular and neuropeptide changes seen in humans and cats. Ann Neurol 33(1):48-56

54. Tepper SJ (2018) History and Review of anti-calcitonin gene-related peptide (CGRP) therapies: from translational research to treatment. Headache. 58(Suppl 3):238-275

55. van den Maagdenberg AM, Pietrobon D, Pizzorusso T, Kaja S, Broos LA, Cesetti T et al (2004) A Cacna 1a knockin migraine mouse model with increased susceptibility to cortical spreading depression. Neuron. 41(5):701-710

56. Boulloche N, Denuelle M, Payoux P, Fabre N, Trotter Y, Géraud G (2010) Photophobia in migraine: an interictal PET study of cortical hyperexcitability and its modulation by pain. J Neurol Neurosurg Psychiatry 81:978-984

57. Denuelle M, Boulloche N, Payoux P, Fabre N, Trotter Y, Geraud G (2011) A PET study of photophobia during spontaneous migraine attacks. Neurology. 76(3):213-218

58. Lai KL, Liao KK, Fuh JL, Wang SJ (2011) Subcortical hyperexcitability in migraineurs: a high-frequency oscillation study. Can J Neurol Sci 38(2): 309-316

59. Granziera C, Daducci A, Romascano D, Roche A, Helms G, Krueger G, Hadjikhani N (2013) Structural abnormalities in the thalamus of migraineurs with aura: a multiparametric study at 3 T. Cerebellum 12(6):812-8

60. Stanimirovic DB, Friedman A (2012) Pathophysiology of the neurovascular unit: disease cause or consequence? J Cereb Blood Flow Metab 32(7):1207-1221

61. Dinia L, Bonzano L, Albano B, Finocchi C, Del Sette M, Saitta L et al (2013) White matter lesions progression in migraine with aura: a clinical and MRI longitudinal study. J Neuroimaging 23(1):47-52 
62. Kruit MC, Launer LJ, Ferrari MD, van Buchem MA (2005) Infarcts in the posterior circulation territory in migraine. The population-based MRI CAMERA study. Brain. 128(Pt 9:2068-2077

63. Schmitz N, Admiraal-Behloul F, Arkink EB, Kruit MC, Schoonman GG, Ferrari $\mathrm{MD}$ et al (2008) Attack frequency and disease duration as indicators for brain damage in migraine. Headache. 48(7):1044-1055

64. Palm-Meinders $\mathrm{IH}$, Koppen $\mathrm{H}$, Terwindt GM, Launer LJ, Konishi J, Moonen JM et al (2012) Structural brain changes in migraine. JAMA. 308(18):1889-1897

65. Gaist D, Garde E, Blaabjerg M, Nielsen HH, Kroigard T, Ostergaard K et al (2016) Migraine with aura and risk of silent brain infarcts and white matter hyperintensities: an MRI study. Brain. 139(Pt 7:2015-2023

66. Granziera C, Romascano D, Daducci A, Roche A, Vincent M, Krueger G, Hadjikhani N (2013) Migraineurs without Aura show microstructural abnormalities in the cerebellum and frontal lobe. Cerebellum 12(6):812-8

67. DaSilva AF, Granziera C, Snyder J, Hadjikhani N (2007) Thickening in the somatosensory cortex of patients with migraine. Neurology. 69(21):1990-1995

68. Granziera C, DaSilva AFM, Snyder J, Tuch DS, Hadjikhani N (2006) Anatomical alterations of the visual motion processing network in migraine with and without aura. PLoS Med 3(10):1915-1921

69. Maleki N, Linnman C, Brawn J, Burstein R, Becerra L, Borsook D (2012) Her versus his migraine: multiple sex differences in brain function and structure. Brain. 135(Pt 8:2546-2559

70. Gaist D, Hougaard A, Garde E, Reislev NL, Wiwie R, Iversen P et al (2018) Migraine with visual aura associated with thicker visual cortex. Brain. 141(3):776-785

71. Hougaard A, Amin FM, Arngrim N, Vlachou M, Larsen VA, Larsson HBW et al (2016) Sensory migraine aura is not associated with structural grey matter abnormalities. Neuroimage Clin 11:322-327

72. Hadjikhani N, Ward N, Boshyan J, Napadow V, Maeda Y, Truini A, Caramia F, Tinelli E, Mainero C (2013) The missing link: enhanced functional connectivity between amygdala and visceroceptive cortex in migraine. Cephalalgia 33(15):1264-8

73. Mainero C, Boshyan J, Hadjikhani N (2011) Altered functional MRI restingstate connectivity in the periaqueductal gray networks in migraine. Ann Neurol 70(2):838-845

74. Burstein R, Cutrer MF, Yarnitsky D (2000) The development of cutaneous allodynia during a migraine attack clinical evidence for the sequential recruitment of spinal and supraspinal nociceptive neurons in migraine. Brain. 123(Pt 8):1703-1709

75. Burstein R, Yarnitsky D, Goor-Aryeh I, Ransil BJ, Bajwa ZH (2000) An association between migraine and cutaneous allodynia. Ann Neurol 47(5):614-624

76. Lipton RB, Bigal ME, Ashina S, Burstein R, Silberstein S, Reed ML et al (2008) Cutaneous allodynia in the migraine population. Ann Neurol 63(2):148-158

77. Bigal ME, Lipton RB (2011) Migraine chronification. Curr Neurol Neurosci Rep 11(2):139-148

78. Doud A, Julius A, Ransom CB (2018) Visual phenomena in occipital lobe epilepsy: "It's Beautiful!!. JAMA Neurol 75(9):1146-1147

79. Walker MC, Smith SJ, Sisodiya SM, Shorvon SD (1995) Case of simple partial status epilepticus in occipital lobe epilepsy misdiagnosed as migraine: clinical, electrophysiological, and magnetic resonance imaging characteristics. Epilepsia. 36(12):1233-1236

80. Panayiotopoulos CP (1999) Visual phenomena and headache in occipita epilepsy: a review, a systematic study and differentiation from migraine. Epileptic Disord 1(4):205-216

81. Kramer DR, Fujii T, Ohiorhenuan I, Liu CY (2017) Interplay between cortical spreading depolarization and seizures. Stereotact Funct Neurosurg 95(1):1-5

82. Ridolfi M, Granato A, Polverino P, Furlanis G, Ukmar M, Zorzenon I et al (2018) Migrainous aura as stroke-mimic: the role of perfusion-computed tomography. Clin Neurol Neurosurg 166:131-135

83. Floery D, Vosko MR, Fellner FA, Fellner C, Ginthoer C, Gruber F et al (2012) Acute-onset migrainous aura mimicking acute stroke: MR perfusion imaging features. AJNR Am J Neuroradiol 33(8):1546-1552

84. Kirshner HS (2011) Transient global amnesia: a brief review and update. Curr Neurol Neurosci Rep. 11(6):578-582

85. Kellner-Weldon F, El-Koussy M, Jung S, Jossen M, Klinger-Gratz PP, Wiest R (2018) Cerebellar Hypoperfusion in migraine attack: incidence and significance. AJNR Am J Neuroradiol. [Epub ahead of print]

Ready to submit your research? Choose BMC and benefit from:

- fast, convenient online submission

- thorough peer review by experienced researchers in your field

- rapid publication on acceptance

- support for research data, including large and complex data types

- gold Open Access which fosters wider collaboration and increased citations

- maximum visibility for your research: over $100 \mathrm{M}$ website views per year

At BMC, research is always in progress.

Learn more biomedcentral.com/submissions 